\title{
ÉCRIRE SANS ECRIRE : BARTHES ET LA RECHERCHE DU ROMAN
}

\author{
por Claudia C. Amigo Pino ${ }^{1}$
}

RÉSUMÉ : Ce texte, présenté comme conférence à l'Université de Bourgogne, à Dijon, le 16 décembre 2008, se propose de réfléchir sur Vita Nova, le roman que Barthes travaillait avant son accident fatal, en février 1980. Pour cela, l'article se centre d'abord sur la description des plans de Vita Nova et des autres textes qui faisaient partie du projet. Ensuite, on entreprend une réflexion sur le projet à partir de trois possibilités d'écriture : 1) Écrire un roman, c'est écrire la Recherche du Temps Perdu ; 2) Écrire un roman, c'est faire le contraire; et 3) Écrire un roman, c'est échapper à la Recherche et tout de même écrire la Recherche.

MOTS CLÉS : Barthes, Vita Nova, Proust, La préparation du roman.

ABSTRACT : First presented as a conference on the $16^{\text {th }}$ of December 2008 at the University of Bourgogne,Dijon, this texts aims at reflecting on Vita Nota, the novel Roland Barthes was writing just before his fatal accident in February, 1980. Thus, in the first part of the article, a description of the plans of Vita Nova and of other texts that were part of the project will be presented. After that, an assessement on the project's three possible ways for writing the novel will be undertaken : 1) Writing the novel is writing Remembrance of Things Past ;2) Writing the novel is doing the exact opposite of what Proust did ; 3) Writing the novel is trying to escape Remembrance of Things Past and, at the same time, rewriting it.

KEYWORDS : Barthes, Vita Nova, Proust, La préparation du roman

RESUMO: Este texto, apresentado como conferência na Université de Bourgogne, em Dijon, França, no dia 16 de dezembro de 2008, propõe uma reflexão sobre Vita Nova, romance que Barthes trabalhava antes de seu acidente fatal, em fevereiro de 1980. Para isso, o artigo se centra primeiramente na descrição dos planos de Vita Nova e dos outros textos que faziam parte do projeto. Em seguida, desenvolveremos uma discussão sobre três possibilidades de escritura : 1) Escrever um romance é escrever Em busca do tempo perdido; 2) Escrever um romance é fazer o contrário; e 3) Escrever um romance é fugir de Em busca do tempo perdido, e mesmo assim escrever Em busca do tempo perdido.

PALAVRAS-CHAVE : Barthes, Vita Nova, Proust, La préparation du roman.

\section{INTRODUCTION : LE DEGRE UN DE L'ECRITURE}

Tout part de zéro. Le premier livre de Barthes, Le degré zéro de l'écriture, de 1953, pose les questions qui se déplieront dans toute son œuvre, parfois diluées, parfois niées et, dans le cas du Barthes des Cours du Collège de France, reprises avec force. A défaut d'une définition transparente et compréhensive de Barthes lui-même (ce qu'il évitait de faire), il nous faudra recréer notre propre zéro, ou faire notre zéro au carré.

Le point zéro pourrait être pensé comme un moment mythique de rencontre du sujet et de l'objet littéraires, le moment où le livre que le lecteur était en train de lire devient le livre que le lecteur va commencer à écrire. Dans ce point précis, il n'y a pas encore d'écriture, en

\footnotetext{
${ }^{1}$ Professeur de Littérature Française à l'Universidade de São Paulo (USP), Brésil, et est auteur du livre A ficção da escrita (publié chez Ateliê Editorial) et Escrever sobre escrever (publié chez Martins Fontes, en 2007, avec Roberto Zular). Elle est coordinatrice du groupe de recherches Criação e Crítica et fait partie du Laboratório do Manuscrito Literário.
} 
fait il n'y a que du silence. Le défi de la critique de Barthes sera décrire cette zone silencieuse, connaître les mécanismes par lesquels elle se produit, et le sens qu'elle produit, son plaisir.

Mais en 1978 il décide publiquement de sortir de ce silence. Son cours au Collège de France porte le nom «La préparation du roman » et, en même temps, il commence à écrire un roman, qui s'appellerait Vita Nova. Dans le cours, il nous renseigne sur ce besoin d'une vie nouvelle, d'où il vient : la souffrance aiguë, due au contact avec la mort, pousse à rompre les liens avec le passé, à passer au volume deux de la vie. Or, changer de vie, selon Barthes, ne pourrait être que changer d'écriture :

Le nouveau escompté, c'est seulement ceci : que la pratique d'écriture rompe avec les pratiques intellectuelles antécédentes; que l'écriture se détache de la gestion du mouvement passé : le sujet écrivant subit une pression sociale pour l'amener (le réduire) à se gérer lui-même, à gérer son œuvre en la répétant : c'est ce ronron qui doit être interrompu.(BARTHES 2003, p.29)

\section{LE PROJET VITA NOVA : PRESENTATION DU MANUSCRIT ET DES ECRITS LIES AU MANUSCRIT}

Quand Barthes est mort accidentellement en 1980, il n'a laissé que quelques feuillets de son roman. Voici la description de l'ensemble de documents faite par Éric Marty, dans les Oeuvres Complètes (Section Écrits Posthumes) :

Vita Nova est composé de huit feuillets de format 21 x 29,7. Seul le huitième est écrit sur un papier quadrillé à petits carreaux, les sept premiers l'étant sur papier type machine à écrire. Cette liasse était rangée dans une chemise cartonnée de couleur rouge sur laquelle était écrit en lettres capitales VITA NOVA. (BARTHES 2002a, p.1007)

Quand on fait référence à ce document, on dit que le contenu des folios est composé par des plans, mot utilisé pour décrire des schémas de récit. Mais je dirai plutôt que ces folios ont l'air d'être des plans : même si une suite est décrite, elle n'est pas une suite d'événements, ni de personnages, ni de déroulements. Il s'agit plutôt d'assemblages et reassemblages d'écrits, ou bien des renvois à des écrits. Parfois ces écrits sont faciles à identifier, parfois ils sont énigmatiques. Il est tout de même important de souligner que dans la plupart des cas, ces écrits existent : ou bien ils étaient en train d'être écrits lorsque Barthes faisait ces plans, ou bien ils étaient déjà écrits. Donc, conclusion évidente : le projet Vita Nova n'est pas composé par huit folios, mais par plusieurs.

Il se peut très bien que ces plusieurs folios du roman Vita Nova soient rien de moins que l'ensemble de textes de Roland Barthes, outre des ouvres d'autres auteurs, comme Pascal, Proust, Tolstoï ou Kafka, pour en citer quelques uns. Comme l'on a déjà remarqué (KNIGHT 1997), ce projet de Barthes a plusieurs points en commun avec Le Livre de Mallarmé et, comme celui-ci, il aura pour toujours le même statut de livre infini.

Cependant, il y a quelques renvois évidents qui peuvent nous aider à avoir l'illusion d'un corpus. D'abord, l'ensemble de notes de La préparation du roman, le deux cours au Collège de France que Barthes a proférés en 1979-1980 dont le sujet était justement la préparation du roman Vita Nova. Cela ajoute presque 500 pages à nos huit folios du départ. En outre, il faut absolument inclure Les fragments d'un discours amoureux, La chambre claire et les notes du cours Le neutre, même s'il reste difficile à dire si Barthes voulait bien 
nier ou affirmer ces projets. De toute façon, ils sont des axes de composition du manuscrit. Finalement, il y a des textes qui faisaient partie du projet et qui, au moins selon ces folios, seraient inclus dans le roman: l'essai «Longtemps je me suis couché de bonne heure », conférence inaugurale au Collège de France en 1979, et les journaux Vaines Soirées, Journal de Deuil, Délibération (Journal de Urt) et Incidents...

On revient aux huit folios initiaux. Les folios sont datés, ce qui ne veut pas dire qu'il les a totalement écrits le jour désigné. C'est probablement la date du début de l'écriture, mais comme ils ont des ajouts et des corrections (à l'encre noire, à l'encre rouge et au crayon), on peut dire que chaque folio est composé par plusieurs couches temporelles. En ce qui concerne ces dates initiales, il est possible d'affirmer que la première écriture du premier folio est datée du 21 août 1979 et que celle du dernier est datée du 12 décembre 1979. Comme Eric Marty l'a décrit, il y a une correspondance entre les sept premiers folios, écrits en papier type machine à écrire. Ces sept folios ont été écrits en octobre et septembre : le huitième est le seul daté de décembre et apparemment il fait partie d'un autre ensemble de notes, d'un autre cahier, ce qui nous fait penser que, même s'il travaillait sur d'autres projets, l'écriture du roman était toujours en train de se faire. Alors seconde conclusion, l'écriture du roman ne s'est pas arrêtée le 12 décembre, même si l'on ne peut préciser une autre date.

\section{POSSIBILITES D’ECRITURE : ENTRE LE DEGRE UN ET LE DEGRE ZERO}

Il est impossible de décrire tous les changements entre les folios ou tous les mouvements d'écriture. Les folios ne sont pas tellement différents les uns des autres, mais les mouvements perceptibles nous mènent à plusieurs réflexions, qu'il serait impossible de développer dans un seul texte. Alors, il faut en choisir quelques unes, moi j'ai choisi notamment celles qui portent sur le rapport avec l'œuvre de Proust, intertexte central dans ce projet.

\section{1 ÉCRIRE UN ROMAN, C'EST RECRIRE LA RECHERCHE}

Dès le début, c'était la Recherche du Temps Perdu. Le premier texte de notre corpus illusoire a comme titre «Longtemps je me suis couché de bonne heure », la phrase initiale de Proust, et bien sûr Barthes l'a choisie parce qu'il voulait prendre cette position de Pierre Ménard des Modernes. Par une coïncidence tragique ou non, son projet n'est jamais devenu un vrai roman, il est resté à l'état de projet, et donc notre premier chapitre doit être le premier texte écrit du projet. Alors, pour les lecteurs d'aujourd'hui, Vita Nova commence effectivement par «Longtemps je me suis couché de bonne heure ».

\section{LE DESIR DE L'AUTEUR}

Cette identification à Proust n'est pas rapportée à un personnage, à une figure du moi, mais à la figure de l'auteur. Après avoir tué l'auteur aux années soixante, Barthes le fait renaître comme un fantôme : en fait, il veut incorporer la démarche du «je » (et pas du «moi ») de la Recherche, autrement dit, il ne s'agit pas d'une identification à un objet du roman, mais à un sujet. 
La recherche est le récit d'un désir d'écrire : je ne m'identifie pas avec à l'auteur prestigieux d'une œuvre monumentale mais à l'ouvrier, tantôt tourmenté, tantôt exalté, de toute manière modeste, qui a voulu entreprendre une tâche à laquelle, dès l'origine de son projet, il a conféré un caractère absolu. (BARTHES 2002a, p. 459)

Comment peut-on décrire cette tâche ? Barthes la regarde de plusieurs points de vue. Le premier et aussi le plus simple est déjà énoncé dans cet extrait qui vient d'être cité : le désir d'écrire. Le roman n'est pas un récit d'une vie, mais le récit du désir d'écrire, alors son roman, Vita Nova, portera sur le même sujet.

\section{L'ECRITURE DU SOMMEIL}

En plus, Barthes aussi fait attention au projet qui se dévoile dans les toutes premières phrases du roman, y compris «Longtemps je me suis couché... ». Pour lui, Proust a voulu écrire sur le sommeil, ou plutôt, à partir du sommeil. Ainsi, il nous fait revenir à l'idée initiale d'identification avec le sujet, avec celui qui énonce le récit, et pas avec le récit. Plus qu'écrire sur le sommeil, Barthes voudrait écrire en dormant, ce qui semble, à première vue, impossible.

Un paradoxe le définit bien : il est un sommeil qui peut être écrit, parce qu'il est une conscience de sommeil ; tout l'épisode (et, partant, je le crois, de toute œuvre qui en sort) se tient ainsi suspendu dans une sorte de scandale grammatical : dire « je dors » est en effet, à la lettre, aussi impossible que de dire «je suis mort »; l'écriture est précisément cette activité qui travaille la langue - les impossibilités de la langue - au profit du discours. (BARTHES 2002a, p. 462)

Il ne s'agit pas d'un sujet nouveau chez Barthes, on pourrait même affirmer qu'une grande partie de son œuvre, comme on l'a déjà dit, dès Le degré zéro de l'écriture jusqu'à Le neutre, en passant par son analyse du conte de Poe «La vérité sur le cas de Monsieur Valdemar », renvoie à ce paradoxe d'écrire là où le sujet disparaît (mort, sommeil). Et cela a toujours été un problème pour lui : comment, pourquoi parler, quand on devrait plutôt se taire $?^{2}$

Dans ce texte, il semble trouver une solution : le narrateur proustien ne renvoie pas au sommeil profond, quand on perd totalement la conscience. Le début de La Recherche porterait sur le bon sommeil d'enfance, ou sur le demi réveil : où l'on entend parfois des bruits, des pas, des bouts de dialogues et on les confond avec la réalité d'un rêve qui vient de commencer, ou bien d'une pensée ou d'un souvenir d'un événement qui s'est passé pendant la journée. Avec cela, Proust aurait fondé une nouvelle logique, la «logique de la vacillation », qui bouleverserait la suite chronologique de la biographie et qui nous ferait rejoindre des ordres inattendus.

\footnotetext{
${ }^{2}$ Je renvois principalement à Le plaisir du texte, où Barthes affirme que « Avec l'écrivain de jouissance (et son lecteur) commence le texte intenable, le texte impossible. Ce texte est hors-plaisir, hors-critique, sauf à être atteint par un autre texte de jouissance : vous ne pouvez parler « sur » un tel texte, vous pouvez seulement parler « en » lui, à sa manière, entrer dans un plagiat éperdu, affirmer hystériquement le vide de la jouissance (et non plus répéter obsessionnellement la lettre du plaisir) ». (BARTHES 2002b, p. 231)
} 


\section{AU DEBUT, LA MORT}

Ce discours du sommeil a deux conséquences pour le projet de roman de Barthes. D'abord, le point de départ du récit : dans tous les plans du roman, on peut remarquer que Vita Nova commencerait aussi de la mort, ou d'un état d'annulation : le deuil, qui occuperait la place d'un «prologue ».

Ce deuil a deux renvois plus au moins explicites. Le plus évident est sans aucun doute la mort de la mère de Barthes, survenue le 25 octobre 1977. Dans les notes de La préparation du roman, il écrit ouvertement que ce serait le motif de l'écriture et du cours sur le roman, ce qui vient avant et pousse l'écriture (un «prologue », justement).

Enfin, un événement, venu du Destin, peut survenir pour marquer, entamer, inciser, articuler, fût-ce douloureusement, dramatiquement, cet ensablement progressif, déterminer ce renversement du paysage trop familier, que j'ai appelé le «milieu du chemin de la vie » : c'est, hélas, l'actif de la douleur. (...) - Pour Proust : la mort de sa mère (1905), même si l'action traumatique, en ce qu'elle produit une mutation active, a lieu plus tard (BARTHES 2003, p. 27-29)

C'est cette douleur intense, ce rapport nouveau avec la mort, qui fait déclencher l'action, dans ce cas «vouloir écrire un roman ». Il est facile de lier cette douleur à la mort de la mère, citée dans la figure de mère de Proust dans cet extrait. Mais dans le manuscrit, il est possible de trouver une autre indication de contact avec la mort et de souffrance intense, le malheur amoureux. Dès le premier plan, on trouve des renvois à ce sujet, juste après le «prologue ». Voici quelques exemples, parmi d'autres :

II. - La décision du 15 avril 1978

- La littérature comme substitut de l'amour

Feuillet 1 (BARTHES 2002a p. 1008)

I. L'acédie amoureuse

- Suite de RH

- Quêtes velléitaires

Feuillet 2 (BARTHES 2002a p. 1009)

On ne sait rien de précis jusqu'à ce moment sur cette décision énigmatique du 15 avril 1978, mais apparemment il s'agit aussi d'un «actif de la douleur », une décision de tout changer, mais qui a affaire plutôt avec une déception amoureuse. Il est très curieux que dans ces cas, on ne peut pas observer une volonté de dépasser la souffrance, de se guérir, mais d'enterrer à jamais l'amour, le substituer par la littérature.

En outre, il faut remarquer l'usage du mot «acédie ». Étant une des figures développées dans son cours Le neutre, l'acédie dans ce cas renvoie à l'absence de désir amoureux, ce qui est aussi le sujet du texte «Vaines soirées » (ici appelé «Quêtes velléitaires »). Finalement, la «suite de RH », nous indique une liaison avec les Fragments d'un discours amoureux: RH sont les initiales d'un ami à Barthes qui apparaissent partout dans le livre et qu'Eric Marty décrit comme la «personne-prétexte » au travers laquelle Barthes a écrit les Fragments (BARTHES 2002a, p.1009). Selon ce texte, le discours 
amoureux est le discours de celui qui n'est pas entendu, et qui ne le sera jamais, parce que l'existence du discours est la constatation de la mort de l'amour :

Savoir qu'on n'écrit pas pour l'autre, savoir que ces choses que je vais écrire ne me feront jamais aimer de qui j'aime, savoir que l'écriture ne compense rien, ne sublime rien, qu'elle est précisément là où tu n'es pas - c'est le commencement de l'écriture. (BARTHES 2002, p. 132)

Alors, l'écriture commence d'un deuil et surtout d'un deuil amoureux : il s'agit toujours de faire un discours pour quelqu'un qui n'est plus là.

\section{LA VACILLATION COMME FORME}

On arrive ainsi à la deuxième conséquence de cette écriture du sommeil pour Vita Nova. Selon les plans, le roman serait composé aussi à partir de cette logique du demi-réveil, ou bien de cette confusion du demi-réveil. Mais à la place des bruits du salon, et des pas de la mère, on entendrait dans ce cas des réflexions, des extraits des essais, les Fragments, des journaux. Autrement dit, ce n'est plus une «vacillation » à entendre, comme celle de Proust, mais une vacillation à lire. Dès le premier plan, on a ces indications de cette pluralité discursive :

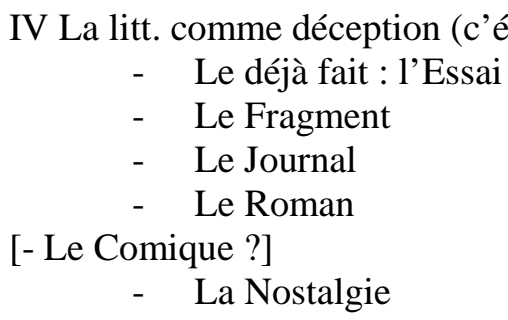

Feuillet 1

Cette notation de forme est plus développée dans les feuillets 6 et 7, qui n'ont pas la forme des «plans » du récit, mais plutôt de notes méta-scripturales sur les formes et les styles du texte. Comme les feuillets sont datés, donc on peut dire que cette idée initiale, qui ne correspond qu'au IV ${ }^{\mathrm{e}}$ moment (ou chapitre) du premier plan, gagne importance avec le cours de l'écriture.

\section{Formes:}

- Le Récit, la relation de quête (intellectuelle) Cf. $\underline{\text { Photo }}$

- La relation de soirée (vanité de la diachronie qui s'étire)

- Les Frgmts d'une " grande cuvre » (cf. Pensées de Pascal) \# observations, aphorismes Frgmts : comme reliefs d'une Apologie de qque chose

- Faux dialogues (serait bon pour le Politique, qui est ratiocination contestatrice sans fin) 
$\mathrm{Au}$ moment où Barthes écrivait ce dernier feuillet, la décision d'incorporer la vacillation était beaucoup plus ambitieuse et était probablement pensée pour la totalité du projet. Il est important de comprendre qu'il ne s'agit pas seulement d'une variation de forme, mais de la « quête du sujet », une sorte de typologie du narrateur (et pourquoi pas, aussi du personnage). Ainsi, dans le récit barthésien, on peut retrouver une quête intellectuelle, une question qui n'arrête pas de se poser et qui doit « rencontrer » une réponse. Dans les Soirées, par contre, la quête est d'un type différent : il s'agit d'étirer le temps le plus possible, même si des questions variées se posent, même si on ne retrouve pas de réponse. Dans les Fragments, il s'agit d'entrevoir une apologie et de faire semblant de ne pas la faire (position très utilisée dans l'ensemble de l'œuvre de Barthes), tandis que dans ces «Faux dialogues », il est question de quelqu'un qui doit toujours contester ce qui est dit.

Dans ces huit feuillets, toutes ces quêtes sont plus au moins éprouvées, mais maintenant il faut se concentrer à la dernière, «Les faux dialogues », puisque elle va nier toutes les autres et va nous mettre dans le fort embarras de revoir tout ce qui était dit auparavant.

\section{2 ÉCRIRE UN ROMAN, C'EST FAIRE LE CONTRAIRE}

\section{LA NOTATION}

Pour commencer cette partie, il faut se poser la question suivante : c'est quoi, faire le contraire de La Recherche?

Si ces genres d'oppositions existaient en littérature, je dirais que ce serait faire un Haïku. Tandis que la Recherche est un de plus grands romans jamais écrits, avec 7 volumes presque sans arrêt (avec très rares pauses de phrase, de paragraphe, ou de chapitre), un pavé solide de mots, le Haïku est un petit poème de 3 vers $(5+7+5)$, un «acte minimal d'énonciation », comme le décrit Barthes. Cette forme de poésie, avec ces tout petits trois vers, n'existe même pas dans la tradition française : il est difficile d'exprimer quoi que ce soit en français avec si peu de mots...

Et pourtant Barthes va dédier son premier séminaire sur le roman, «La préparation du roman $1 »$ (de Décembre 78 à Mars 79), ce roman qui serait une réécriture de la Recherche, à une réflexion sur le Haïku.

Barthes justifie ce choix très bizarre pour parler de l'écriture du roman avec une réflexion sur le rapport au passé, son passé. Pour lui, son roman fantasmé devrait effacer la mémoire, «ne pas raconter ce qui ne reviendra plus »(BARTHES 2003, p.45). Mas comment échapper au passé dans un récit ? Barthes se propose d'utiliser la notation comme base, la notation du présent. Le Haïku serait exactement une forme établie, contestée, renouvelée, des notations du présent.

Les renvois déjà cités aux journaux, aux Soirées, aux Fragments, ont carrément affaire avec cette contrainte de la notation du présent et de l'esthétique du Haïku. En plus, il faut citer aussi un autre texte qui apparaît dans presque tous les feuillets, mais qui n'est pas explicitement cité : Incidents (écrit en 1969-70 et publié posthumément par François Wahl en 1987), des notations d'un voyage au Maroc et dont on reviendra plus tard. 


\section{LA VRAIE VERITE}

Aucun Haïku n'est cité dans le manuscrit, ce qui est un peu énigmatique. Il est important de remarquer que ces huit feuillets sont en fait très postérieurs au cours, qui avait commencé en décembre 1978 et qui a été probablement écrit avant cette date. Peut-être cela est du fait que toutes ces tentatives de notation du présent sont ratées par rapport au Haïku. Les journaux ne sont pas des caméras, ils n'enregistrent pas le présent, il s'agit toujours d'un récit d'un moment déjà fini, autrement dit, une écriture du passé. Cela peut nous faire croire que le manuscrit a pris un autre chemin, toutefois, le Haïku persiste, mais plutôt comme un problème moral.

Dans le cours du Haïku, Barthes pose petit à petit ce problème moral, le problème de la vérité. Voici un des premiers moments où il touche ce problème, à propos de la lecture des traductions des Haïkus :

Le haïku m'est humain, absolument humain. Comment cela est-il possible (pour moi jamais cette sensation de familiarité avec d'autres poésies traduites) ?

Je me l'explique ainsi : le haïku est la conjonction d'une «vérité » (non conceptuelle, mais de l'Instant) et d'une forme. Je pense à cet autre mot de Valéry : «... faire voir que la pensée pure et la découverte de la vérité en soi ne peuvent jamais aspirer qu'à la découverte ou à la construction de quelque forme » : oui, je le crois, la forme («quelque forme ») prouve, manifeste la vérité (et non pas seulement le « raisonnement»). (BARTHES, 2003, p. 5556)

Roland Barthes aspire à écrire un roman qui dise aussi cette vérité non conceptuelle, mais formelle. Plus tard, il dira qu'elle n'est pas représentative non plus, qu'il ne s'agit pas d'un discours sur la vérité, mais la vérité même, «la chose même atteinte par l'Affect »: «Amour et Mort sont là, c'est tout ce qu'on peut dire. Et c'est le mot même du haïku ». (BARTHES, 2003, p. 159)

Il y a deux genres de notations dans le manuscrit qui renvoient à cette vérité affective que le roman devrait produire. D'abord, l'idée que la littérature devrait être un substitut de l'amour, présente dès le premier feuillet au second chapitre ou seconde partie, comme l'on a déjà vu. En outre, on doit citer le mystérieux épilogue avec lequel le roman devrait finir au moins selon les trois premiers plans :

Epilogue : la Rencontre (BARTHES, 2002a, p. 1010-1012)

Si on lie cet épilogue à la décision de substituer la littérature à l'amour, il me semble que la fin du livre donnerait (même si l'on ne sait pas à qui, au narrateur ou au lecteur) cette sensation de « rencontre amoureuse » littéraire. Autrement dit, la fin serait l'accomplissement de la quête initiale de la Vita Nova.

Mais l'écriture chemine vers le contraire. L'épilogue apparaît dans la même place, avec les mêmes mots, à la fin du premier et second plan. Au troisième plan, il est mis en question : on le retrouve précédé d'un signe d'interrogation et suivie d'index, ce qui veut dire que le texte ni finirait pas avec cette rencontre :

\section{? Epilogue La Rencontre}


Dans les prochains plans, l'épilogue simplement disparaît et la décision de substituer l'amour à la littérature se déplace petit à petit jusqu'à arriver à la fin, ce qui nous mène à penser qu'elle ne serait plus une quête du roman, mais une tâche à l'avenir. Je cite la forme comme elle apparaît au cinquième feuillet, qui peut résumer cette tendance d'écriture :

Décision du 15 avril 1978

$-\mathrm{VN}$

- « Je me retire pour entreprendre une grande œuvre

où serait dit... l'Amour

Feuillet 5 (BARTHES, 2002a, p. 1015)

D'abord, il faut remarquer que cette disposition commence par des guillemets qui seront ouverts à jamais, ce qui nous pose vraiment dans l'avenir. Outre cela, celui qui écrit se retire pour entreprendre une grande œuvre, mais paradoxalement, il se retire précisément de cette œuvre où il songeait de dire l'amour. D'une part, on peut remarquer le rapport avec la fin de la Recherche, où le narrateur se rend compte du besoin d'écrire l'œuvre que, en fait, il est en train de terminer. Par ailleurs, ce n'est pas tout à fait la même situation, parce qu'ici le scripteur se retire pour entreprendre une autre œuvre, qui apparemment n'est pas celle que le lecteur est en train (ou sera en train) de lire.

\section{NE PAS ECRIRE UN ROMAN}

A la conférence inaugurale du cours, il était question déjà d'écrire un roman et en même temps de trouver une autre forme, une «tierce forme » entre l'essai et le roman. Alors, dès son départ, le projet de la préparation du roman incluait aussi sa destruction : il était question aussi de ne pas écrire un roman.

On a déjà vu que ce refus du roman a comme base le refus du récit du passé, du lien avec ce qui ne reviendra pas. Mais ce dernier point, sur le besoin de dire la vérité, nous fait aussi penser à ce refus de la démarche que l'auteur est en train d'entreprendre. Roland Barthes, au milieu de son cours, se rend compte de son propre refus à son projet et, au contraire de le cacher pour être cohérent, il décide de l'explorer.

Quand je produis des Notations, elle sont toutes «vraies » : je mens jamais (je n'invente jamais), mais précisément je n'accède pas au Roman : le roman commencerait non au faux, mais quand on mêle sans le prévenir le vrai et le faux : le vrai absolu et le faux colorié, brillant, venu de l'ordre du Désir et l'Imaginaire. (...)

Peut-être donc : parvenir à faire un roman (telle est la perspective) - le point de fuite - de notre cours), c'est au fond d'accepter de mentir (ce peut être très difficile, de mentir) - mentir de ce mensonge second et pervers qui consiste à mêler le vrai et le faux $\rightarrow$ En définitive, alors, la résistance au roman, l'impuissance au roman (à sa pratique) serait une résistance morale. (BARTHES, 2003, p. 161)

La découverte de cette résistance morale au roman est aussi présente dans le manuscrit de Vita Nova, de plusieurs façons. D'abord, au septième feuillet, on retrouve une allusion à ce besoin de ne pas faire semblant d'être un autre, de ne pas inventer des personnages, des mois menteurs, des faux : 
- Renoncer au jeu s/la Bêtise, les guillemets, les refus de prendre position sur l'énonciation (alibi du

Romanesque, de la diversité de mon moi).

Sans Complaisance. Pas de Semblant.

Feuillet 7 (BARTHES, 2002a, p. 1016)

Mais on arrive plus loin. Il ne renonce qu'aux guillemets, en divers moments il affirme ouvertement (mais pourquoi l'écrire ? Si c'était vrai, il aurait suffit de jeter tout à la poubelle) qu'il n'écrirait pas le roman, qu'il n'aurait plus une Vita Nova:

- Tout ceci voudrait dire qu'on abandonne l'enfantillage du Récit Vita Nova : ces efforts de grenouille qui veut se faire aussi grosse...

Feuillet 7 (BARTHES, 2002a, p. 1017)

Tout cela en fait revient à zéro, revient à ce mythique premier livre de Barthes, où il était question d'introduire le moment de rencontre du sujet et de l'objet littéraires. Voilà Roland Barthes de nouveau tombé sur le silence, sur le besoin impératif de ne pas écrire. Il s'est rendu compte que les efforts de construire un récit, ce sont des efforts pour faire du semblant, pour mentir, pour se déguiser. La seule façon de produire la vérité qu'il est en train de chercher est de produire ce silence à deux, du lecteur et de l'auteur. Il s'est rendu compte que lui-même, Roland Barthes, il est arrivé à ce silence, il voit qu'il doit amener le lecteur à la même place et ce ne sera que par une voie : lui faire sentir la même déception qu'il a subi.

\section{3 ÉCRIRE UN ROMAN, C'EST ECHAPPER A LA RECHERCHE ET TOUT DE MEME ECRIRE LA RECHERCHE}

Pour commencer cette dernière partie, il faut remarquer un point tout à fait important: Roland Barthes n'a jamais écrit quoi que ce soit qui puisse ressembler à la Recherche. Ce texte n'est pas écrit, c'est un texte à faire et, en plus, ce n'est pas lui qui est en train de le faire.

Dès le premier plan et partout dans le manuscrit on peut trouver des renvois à l' « oisiveté », ou bien à l' "enfant marocain ». Cet enfant, cité dans Incidents, illustre ce que Roland Barthes voulait dire avec « oisiveté », cette activité de ne rien faire, de ne pas bouger, de ne pas avoir une « quête »:

Un gosse assis sur un mur bas, au bord de la route qu'il ne regarde pas -assis comme éternellement, assis pour être assis, sans tergiverser :

«Assis paisiblement sans rien faire,

Le printemps vient et l'herbe croit d'elle-même. » (BARTHES, 2002a, p. 974)

Plus que m'arrêter sur la définition d' « oisiveté $»^{3}$, je voudrais juste rappeler un commentaire que Barthes fait pendant le cours sur le roman. Au moment où il introduit ce concept et cet enfant marocain, il ouvre une parenthèse :

\footnotetext{
${ }^{3}$ Ce qui a été très bien fait par Diana Knight dans son article «Idle thoughts : Barthes's Vita Nova ».
} 
(M'enchante l'anacoluthe : il n'y a plus de sujet rien qu'un «Assis »]. J'ai vécu absolument cet état, non hélas par moi-même - c'est bien là le problème - mais par procuration, en voyant, un jour où je conduisait seul l'auto, lentement, sur une route très secondaire du Maroc, vers Ben Slimane, un enfant assis sur un vieux mur - et c'était le printemps. (BARTHES, 2003, p. 216-217)

Barthes était enchanté de l'absence de sujet, ou bien de la dissolution de ce sujet. Par contre, l'histoire racontée a deux sujets : Barthes lui-même, qui conduisait seul, et l'enfant, qui était assis à côté de la route. Si celui-ci ne faisait rien, s'il était dans cet état d'oisiveté, on n'en sait rien, cet état fut le produit de la rencontre entre sujet et objet, entre celui qui était en quête (celui qui conduisait) et celui qui était à côté, sans regarder cette route.

Cette petite anecdote peut être prise comme une fable sur l'écriture du roman. Bien sûr, il faut avoir une quête, il faut explorer le désir d'écrire, comme Proust. Voilà pourquoi il faut toujours écrire la Recherche. Mais, en même temps, pour arriver à cette vérité envisagée, il faut échapper à la Recherche, lâcher son désir d'écriture, ne plus être Proust, être quelqu'un d'autre, par procuration. Cette possibilité d'être un autre ne sera pas du mensonge, du semblant, ce serait vraiment dire...l'Amour.

Comment laisser d'être ce Roland Barthes et sa volonté d'écrire un roman, comment avoir cette procuration qui permettrait d'être un autre sans mentir?

Roland Barthes est mort en mars 1980. Si l'on ne retrouve pas par hasard un mystérieux tapuscrit de roman achevé, peut être on ne le saura jamais. Mais on peut esquisser une hypothèse : la seule façon d'avoir cette procuration, ce serait de disparaître, de laisser comme testament un manuscrit inachevé, le publier comme texte posthume aux ouvres complètes, faire surgir partout dans le monde des lecteurs inquiets, qui voudraient « reconstruire » l'écriture de cette œuvre et qui, pour cela, devront se mettre à la place de l'auteur, être quelqu'un d'autre, par procuration...

\section{BibLIOGRAPHIE :}

BARTHES, Roland. La Préparation du Roman I et II. Cours et séminaires au Collège de France (1978-1979 et 1979 -1980) Texte établi, annoté et présenté par Nathalie Léger. Paris : Seuil/IMEC, 2003.

. Oeuvres Complètes. Vol. V. Livres, Textes, Entretiens. 1977-1980. Nouvelle éd. revue, corrigé et présentée par Éric Marty. Paris : Seuil, 2002a, p. 1007

. Oeuvres Complètes. Vol. IV. Livres, Textes, Entretiens. 1977-1980. Nouvelle éd. revue, corrigé et présentée par Éric Marty. Paris : Seuil, 2002b, p. 1007

KNIGHT, Diana. "Idle thoughts: Barthes's Vita Nova". Nottingham French Studies. Vol. 36, Spring, 1997. 